\section{REFERENCES}

1. Bowe WP, Patel N, Logan AC. Acne vulgaris: the role of oxidative stress and the potential therapeutic value of local and systemic antioxidants. J Drugs Dermatol 2012;11:742-746.

2. Sahib AS, Al-Anbari HH, Raghif ARA. Oxidative stress in acne vulgaris: an important therapeutic target. J Mol Pathophysiol 2013;2:27-31.

3. Kovalev VM. [Complex treatment of acne with lipoic acid]. Vrach Delo 1981;(3):108-113. Russian.

4. Li X, Yang Z, Bai Y. Fluorescence spectroscopic analysis of the interaction of papain and bromelain with I-ascorbic acid, a-tocopherol, $\beta$-carotene and astaxanthin. Int J Biol Macromol 2018;107:144-156.

5. Choi CW, Choi JW, Park KC, Youn SW. Facial sebum affects the development of acne, especially the distribution of inflammatory acne. J Eur Acad Dermatol Venereol 2013; 27:301-306.

6. Chang TM, Tsen JH, Yen H, Yang TY, Huang HC. Extract from periostracum cicadae inhibits oxidative stress and inflammation induced by ultraviolet $\mathrm{B}$ irradiation on $\mathrm{HaCaT}$ keratinocytes. Evid Based Complement Alternat Med 2017; 2017:8325049.

7. Bromfield JJ, lacovides SM. Evaluating lipopolysaccharideinduced oxidative stress in bovine granulosa cells. J Assist Reprod Genet 2017;34:1619-1626.

8. Ganesan K, Tiwari M, Balachandran C, Manohar BM, Puvanakrishnan R. Estrogen and testosterone attenuate extracellular matrix loss in collagen-induced arthritis in rats. Calcif Tissue Int 2008;83:354-364.

9. Ripple MO, Henry WF, Rago RP, Wilding G. Prooxidantantioxidant shift induced by androgen treatment of human prostate carcinoma cells. J Natl Cancer Inst 1997;89:40-48.

10. Lee WJ, Kim SL, Choe YS, Jang YH, Lee SJ, Kim DW. Magnesium ascorbyl phosphate regulates the expression of inflammatory biomarkers in cultured sebocytes. Ann Dermatol 2015;27:376-382.

\title{
Plasmacytoid Dendritic Cells in Pityriasis Rubra Pilaris
}

\author{
Jana Al-Hage*, Randa Akel*, Mazen Kurban, Ossama Abbas \\ Department of Dermatology, American University of Beirut Medical Center, Beirut, Lebanon
}

\section{Dear Editor:}

Plasmacytoid dendritic cells (pDCs) are bone marrow-derived DCs with plasma cell morphology. They are lineage negative and express CD4, CD123, HLA-DR, blood-derived dendritic cell antigen-2 (BDCA-2) and Toll-like receptor (TLR) 7 and TLR9 within endosomal compartments ${ }^{1}$. When activated, TLRs initiate a cascade of multiple signaling pathways that ultimately lead to production of pro-inflammatory cytokines such as type I interferons (INFs) and, to a lesser extent, IL- 6 and TNF- $\alpha$. This cytokine profile, especially type I IFN, is responsible for pDCs' anti-viral effect as well as their ability to link innate and adaptive immunity ${ }^{1}$. In addition to their role in cancer immunity and cutaneous viral infections, pDCs have pathogenetically been implicated in several inflammatory skin diseases such as lupus erythematosus (LE), psoriasis, lichen planus, alopecia areata, among others ${ }^{1}$.

Pytiriasis rubra pilaris (PRP) is a rare idiopathic papulosqu-

Received October 25, 2017, Revised January 24, 2018, Accepted for publication February 23, 2018

Corresponding author: Ossama Abbas, Department of Dermatology, American University of Beirut Medical Center, Riad El Solh St., P.O.Box 11-0236, Beirut 11072020, Lebanon. Tel: 961-1-350000, ext. 7915, Fax: 961-1-745320, E-mail: ossamaabbas2003@yahoo.com ORCID: https://orcid.org/0000-0001-6970-8056

*These authors have equally contributed to the article.

This is an Open Access article distributed under the terms of the Creative Commons Attribution Non-Commercial License (http://creativecommons.org/ licenses/by-nc/4.0) which permits unrestricted non-commercial use, distribution, and reproduction in any medium, provided the original work is properly cited.

Copyright (C) The Korean Dermatological Association and The Korean Society for Investigative Dermatology 
amous inflammatory skin disorder, with heterogeneous clinical manifestations, characterized by follicular keratosis, orange-red scaling plaques with "Islands of sparing" and palmoplantar hyperkeratosis ${ }^{2}$. PRP shares with psoriasis many overlapping features. The presence of a focally thickened stratum granulosum, follicular plugging and foci of acantholysis are helpful tools in favoring $\mathrm{PRP}^{2}$. PRP pathogenesis is still poorly understood. The role of abnormal vitamin A metabolism in the skin with disruption of the retinoid signaling pathways has long been considered as a potential inducer of increased epidermal proliferation ${ }^{2}$. Genetics also play a role in PRP. An autosomal dominant familial type was recently demonstrated with mutations in CARD14 gene ${ }^{3}$. This gene is also implicated in familial psoriasis, making familial PRP a possible allelic variant to familial psoriasis ${ }^{3}$. Rarely, CARD14 genetic mutations have also been demonstrated in sporadic PRP cas$\mathrm{es}^{3}$. Given the overlapping clinicopathological and genetic features between PRP and psoriasis and since pDCs have been implicated in psoriasis pathogenesis, we intend to investigate any role of pDCs in PRP, which has not been studied before.

Our institutional review board approved the study (IRB no. DER.OA.12). Twenty one PRP and 18 psoriasis vulgaris cases were retrieved from our database. Only straightforward cases were included. None of the patients was receiving treatment when the biopsy was performed. Immunohistochemical analysis was performed on sections

Table 1. pDC presence and MxA expression in PRP and psoriasis (\%)

\begin{tabular}{|c|c|c|c|c|c|c|c|c|}
\hline \multirow{2}{*}{ Entity } & \multirow{2}{*}{$\begin{array}{l}\text { pDC infiltration } \\
\text { (n) }\end{array}$} & \multicolumn{4}{|c|}{ pDC score } & \multicolumn{3}{|c|}{ MxA score } \\
\hline & & 0 & 1 & 2 & 3 & 0 & 1 & 2 \\
\hline $\operatorname{PRP}(n=21)$ & $18 / 21$ & 3 & 17 & 1 & 0 & 3 & 14 & 4 \\
\hline Psoriasis $(n=18)$ & $18 / 18$ & 0 & 6 & 8 & 4 & 0 & 14 & 4 \\
\hline$p$-value* & $>0.05$ & \multicolumn{4}{|c|}{$<0.05$} & \multicolumn{3}{|c|}{$>0.05$} \\
\hline
\end{tabular}

pDC: plasmacytoid dendritic cell, MxA: myxovirus protein A, PRP: pityriasis rubra pilaris. * Statistical analysis was performed by using the Mann-Whitney test to analyze statistical differences in pDC andbetween the 2 entities. A two-tailed $p$-value of $<0.05$ isconsidered statistically significant.
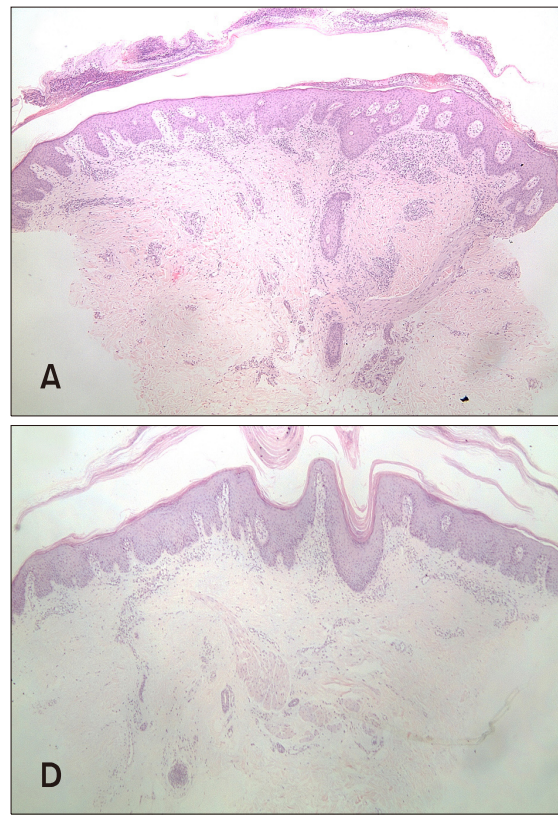
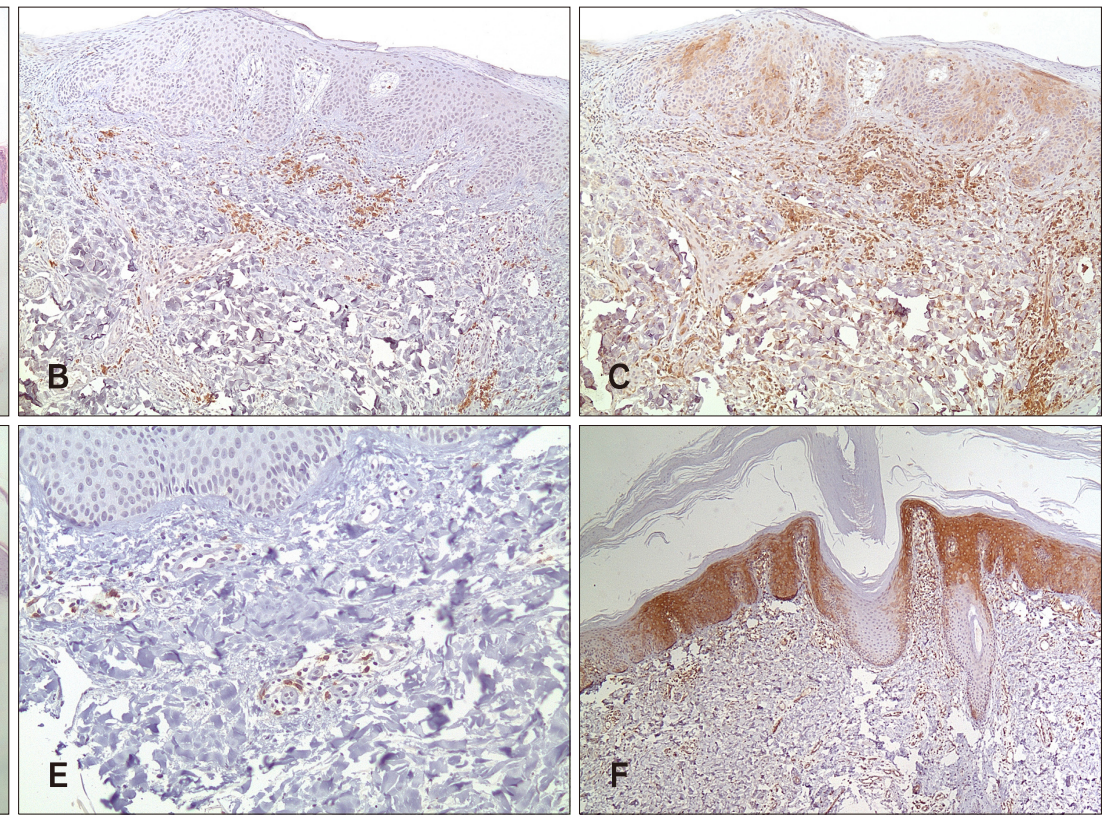

Fig. 1. (A $\sim C$ ) Psoriasis. (A) Representative case showing classical microscopic features of psoriasis $(H \& E, \times 40)$. (B) Blood-derived dendritic cell antigen-2 (BDCA-2) immunostaining highlighted plasmacytoid dendritic cells (pDCs) in a superficial perivascular distribution $(\times 100)$. (C) Patchy myxovirus protein $A(M \times A)$ immunostaining of both the epithelium and inflammatory cells $(\times 100)$. (D F) PRP. (D) Representative case showing alternating ortho- and parakeratosis, follicular plugging, psoriasiform acanthosis and underlying dermal inflammatory infiltrate $(\mathrm{H} \& \mathrm{E}, \times 40)$. (E) BDCA-2 immunostaining highlighted pDCs in a superficial perivascular distribution ( $\times 200)$. (F) Patchy MxA immunostaining of both the epithelium and inflammatory cells $(\times 100)$. 
obtained from formalin-fixed, paraffin-embedded tissue using antibodies to BDCA-2 (mouse immuno-globulin G1, clone 124B3.13; Dendritics, Lyon, France) and myxovirus resistance protein $\mathrm{A}(\mathrm{M} \times \mathrm{A}, \mathrm{M} 143$; University of Freiburg, Freiburg, Germany, Professor Haller). Anti-BDCA2 antibody is a specific pDC marker ${ }^{1}$, while anti-MxA antibody assesses type I IFN production by pDCs, since MxA is well established surrogate marker for local type I IFN production ${ }^{1}$. A semiquantitative scoring system was used to assess pDC recruitment and MxA expression. pDCs content was scored as percentage of the total mononuclear infiltrate: 0 (absent or very rare positive cells), $1(1 \% \sim 10 \%$ of positive cells), 2 (10\% $\sim 50 \%$ of positive cells), 3 (> $>50 \%$ of positive cells). MxA staining was scored as: 0 (negative), 1 (weak/patchy staining), and 2 (intense/diffuse staining). Normal skin tissue served as negative control (pDC score of 0, MxA score of 0 ) and discoid LE served as positive control (pDC score of 3, MxA score of 2) (Table 1).

PRP patients (13 men and 8 women; 14 type I and 7 type III) ranged in age from 6 to 69 years (mean of 39). Disease onset prior to biopsy ranged between 1 to 4 weeks. Psoriasis patients (8 men and 10 women) had all psoriasis vulgaris and ranged in age from 10 to 72 years (mean of 44 years). Disease onset ranged between 3 and 9 weeks. Results (Fig. 1) showed the pDCs to be present in 18/21 (86\%) PRP cases and 18/18 (100\%) psoriasis cases and pDCs were significantly less abundant in PRP than in psoriasis cases. Patchy weak MxA expression was similarly present in both PRP and psoriasis cases.

Our hypothesis in this study concerning pDC role in PRP pathogenesis is based on several observations. First, PRP has been reported in association with different viral infections such as herpes virus infections, hepatitis $A$ and $C$, and HIV, which is associated with type VI PRP ${ }^{4,5}$. It is not surprising that pDCs may be implicated in such immune pathways, since they are known to be key effectors in innate antiviral immunity ${ }^{1}$. Second, PRP has been associated with several inflammatory disorders such as LE, lichen planus, alopecia areata and vitiligo ${ }^{5,6}$, in which evidence suggests significant pDC role in their underlying pathogenesis ${ }^{1}$. Third, imiquimod, an immunomodulator known to be a potent pDC activator through its effect on TLR-7, has been reported to induce or exacerbate PRP ${ }^{7}$. Fourth, PRP relation to psoriasis may raise the possibility of similar underlying pathogenesis. In addition to the genetic and clinicopathological overlap between PRP and psoriasis, recent evidence suggests shared pathogenic pathways $^{8}$. Similar to psoriasis, a recent study identified a role of the IL-23-TH17-axis in $\mathrm{PRP}^{8}$. In the study, upregulated expression of most proinflammatory innate cytokines (TNF, IL-6, IL-12, IL-23, and IL-1 $\beta$ ) and adaptive T-cell cy- tokines such as TH1 and, especially, TH17 (IL-17A, IL-17F, and IL-22) cytokines was demonstrated in lesional PRP skin lesions. Clinical and histopathologic improvement of PRP lesions in one patient treated with ustekinumab (human anti-IL-12/IL-23 antibody) paralleled the expression levels of $\mathrm{TH} 17$ cytokines, further supporting the role of IL-23-TH17-axis in PRP. Activated pDC and type I IFNs are now obviously implicated in the initial innate immune events that drive inflammation in psoriasis ${ }^{9}$. pDCs infiltrate early psoriatic skin lesions in correlation with expression of markers typical of early psoriasis phases ${ }^{9}$. In psoriatic skin, extracellular self-DNA fragments get coupled to overexpressed endogenous antimicrobial peptides. This breaks innate tolerance via TLR9 activation and leads to pDC activation and IFN production ${ }^{9}$. In addition, pDC relation to IL-23-TH17-axis, implicated in psoriasis and PRP, has recently been partially clarified. A recent study demonstrated that imiquimod-stimulated pDCs can initiate Th17-deviated inflammation in humans, which partially explains reports of imiquimod inducing or exacerbating psoriasis and $\mathrm{PRP}^{10}$.

In conclusion, we have demonstrated pDC presence as part of the inflammatory infiltrate in most PRP cases suggesting possible role of pDCs in PRP, possibly in the initial steps of disease onset similar to psoriasis.

\section{CONFLICT OF INTEREST}

The authors have nothing to disclose.

\section{ORCID}

Jana Al-Hage, https://orcid.org/0000-0001-7506-527X

Randa Akel, https://orcid.org/0000-0001-8028-8830

Mazen Kurban, https://orcid.org/0000-0003-1011-0687

Ossama Abbas, https://orcid.org/0000-0001-6970-8056

\section{REFERENCES}

1. Saadeh D, Kurban M, Abbas O. Update on the role of plasmacytoid dendritic cells in inflammatory/autoimmune skin diseases. Exp Dermatol 2016;25:415-421.

2. Marrouche N, Kurban M, Kibbi AG, Abbas O. Pityriasis rubra pilaris: clinicopathological study of 32 cases from Lebanon. Int J Dermatol 2014;53:434-439.

3. Takeichi T, Sugiura K, Nomura T, Sakamoto T, Ogawa Y, Oiso $N$, et al. Pityriasis rubra pilaris type $V$ as an autoinflammatory disease by CARD14 mutations. JAMA Dermatol 2017;153:66-70.

4. Wang T, Liu J, Liu Y, Zheng H. Pityriasis rubra pilaris (PRP) with preceding Epstein-Barr virus infection: a new type PRP with non-HIV virus infection? Chin Med J (Engl) 2014;127:391. 
Brief Report

5. Cecchi R, Giomi A, Tuci F, Bartoli L, Seghieri G. Pityriasis rubra pilaris, Lichen planus, Alopecia universalis and vitiligo in a patient with chronic viral hepatitis $\mathrm{C}$. Dermatology 1994;188:239-240.

6. Lerner MR, Braverman IM. Psoriasis, lupus erythematosus, and Pityriasis rubra pilaris: occurrence in one family. Arch dermatol 1962;85:229-231.

7. Atanaskova Mesinkovska N, Dawes D, Sood A, Bergfeld W. Acantholytic Pityriasis rubra pilaris associated with imiquimod $3.75 \%$ application. Case Rep Dermatol Med 2011;2011:412684.

8. Feldmeyer L, Mylonas A, Demaria O, Mennella A,
Yawalkar N, Laffitte E, et al. Interleukin 23-helper T cell 17 axis as a treatment target for Pityriasis rubra pilaris. JAMA Dermatol 2017; 153:304-308.

9. Lande R, Chamilos G, Ganguly D, Demaria O, Frasca L, Durr $\mathrm{S}$, et al. Cationic antimicrobial peptides in psoriatic skin cooperate to break innate tolerance to self-DNA. Eur J Immunol 2015;45:203-213.

10. Garzorz-Stark N, Lauffer F, Krause L, Thomas J, Atenhan A, Franz $R$, et al. Toll-like receptor $7 / 8$ agonists stimulate plasmacytoid dendritic cells to initiate a TH17-deviated acute contact dermatitis in human subjects. J Allergy Clin Immunol 2018;141:1320-1333.e11.

\title{
Incidence of Topical Tacrolimus Adverse Effects in Chronic Skin Disease
}

\author{
Hyung-jin Park, Min-jae Gwak, Ki-Heon Jeong, Mu-Hyoung Lee, Min Kyung Shin \\ Department of Dermatology, School of Medicine, Kyung Hee University, Seoul, Korea
}

\section{Dear Editor:}

Tacrolimus ointment is approved by the Food and Drug Administration (FDA) for second-line therapy of moderate to severe atopic dermatitis therapy. There are other indications for topical tacrolimus, including contact dermatitis, psoriasis, seborrheic dermatitis, vitiligo, and rosacea ${ }^{1}$. Common adverse effects (AEs) include application-site reactions, such as burning sensation, pruritus, and erythema. The incidence of burning sensation after application of $0.1 \%$ tacrolimus was $19 \% \sim 59 \%$, followed by pruritus. However, these are generally transient and have mild to moderate intensity. Primary disease status improvement is associated with relief of $\mathrm{AEs}$, and $\mathrm{AE}$-associated treatment discontinuation is rarely reported. The percentage of patients prematurely discontinuing treatment because of $\mathrm{AES}$ was $1.4 \% \sim 6.2 \%$ with $0.03 \%$ tacrolimus ointment and $1.6 \% \sim 5.3 \%$ with $0.1 \%$ tacrolimus ointment ${ }^{2}$.

Topical tacrolimus is relatively safe, but patient compliance can be impacted by AEs at the beginning of treatment. Additional studies are needed to determine whether the incidence of these AEs differ by disease conditions or applied site. Therefore, we conducted a retrospective chart review to assess the incidence patterns.

Data from patients prescribed topical tacrolimus (Protopic ${ }^{\circledR}$; Astellas Pharma, Tokyo, Japan) from March 2012 to November 2014 were collected retrospectively. Five common diseases treated with topical tacrolimus in our hospital were identified, and these diseases were commonly known to involve the neurogenic pathway in pathogenesis to some extent. AEs such as burning sensation, pruritus,

\footnotetext{
Received November 30, 2017, Revised January 16, 2018, Accepted for publication March 7, 2018
}

Corresponding author: Min Kyung Shin, Department of Dermatology, School of Medicine, Kyung Hee University, 23 Kyungheedae-ro, Dongdaemun-gu, Seoul 02447, Korea. Tel: 82-2-958-8300, Fax: 82-2-969-6538, E-mail: haddal@hanmail.net ORCID: https://orcid.org/0000-0001-9834-7931

This is an Open Access article distributed under the terms of the Creative Commons Attribution Non-Commercial License (http://creativecommons.org/ licenses/by-nc/4.0) which permits unrestricted non-commercial use, distribution, and reproduction in any medium, provided the original work is properly cited.

Copyright (C) The Korean Dermatological Association and The Korean Society for Investigative Dermatology 